\title{
creative
commons
}

ISSN 1855-3966 (printed edn.), ISSN 1855-3974 (electronic edn.)

ARS MATHEMATICA CONTEMPORANEA 18 (2020) 105-115

https://doi.org/10.26493/1855-3974.1933.2df

(Also available at http://amc-journal.eu)

\section{Notes on exceptional signed graphs}

\author{
Zoran Stanić * \\ Faculty of Mathematics, University of Belgrade, \\ Studentski trg 16, 11000 Belgrade, Serbia
}

Received 14 February 2019, accepted 25 December 2019, published online 24 September 2020

\begin{abstract}
A connected signed graph is called exceptional if it has a representation in the root system $E_{8}$, but has not in any $D_{k}$. In this study we obtain some properties of these signed graphs, mostly expressed in terms of those that are maximal with a fixed number of eigenvalues distinct from -2 . As an application, we characterize exceptional signed graphs with exactly 2 eigenvalues. In some particular cases, we prove the (non-)existence of such signed graphs.
\end{abstract}

Keywords: Adjacency matrix, least eigenvalue, root system, signed line graph, exceptional signed graph, signed graph decomposition.

Math. Subj. Class. (2020): 05C22, 05C50

\section{Introduction}

A signed graph $\dot{G}$ is a pair $(G, \sigma)$, where $G=(V, E)$ is a finite unsigned graph, called the underlying graph, and $\sigma: E \longrightarrow\{-1,1\}$ is the signature. The edge set of a signed graph is composed of subsets of positive and negative edges. Throughout the paper we interpret a graph as a signed graph with all the edges being positive.

The $n \times n$ adjacency matrix $A_{\dot{G}}$ of $\dot{G}$ is obtained from the $(0,1)$-adjacency matrix of $G$ by reversing the sign of all $1 \mathrm{~s}$ which correspond to negative edges. The eigenvalues of $A_{\dot{G}}$ are real and form the spectrum of $\dot{G}$.

We establish some properties of exceptional signed graphs. Precisely, we extend some results concerning the particular case of exceptional graphs, which can be found in [3]. It occurs that the 'signed' generalizations of some known theorems have a nice form and give an interesting insight into the nature of signed graphs. We also give a characterization

* This research is partially supported by Serbian Ministry of Education, Science and Technological Development, Projects 174012 and 174033.

E-mail address: zstanic@math.rs (Zoran Stanić)

(ㄷ) This work is licensed under https://creativecommons.org/licenses/by/4.0/ 
of exceptional signed graphs with 2 (distinct) eigenvalues in terms of certain decompositions which are specified in the corresponding section. Some particular exceptional signed graphs with 2 eigenvalues are determined.

Sections 2 and 3 are preparatory. In the former we fix some terminology and notation, and in the latter we give a brief review of the root systems with a special emphasis on $D_{k}$ and $E_{8}$. Our main contribution is reported in Sections 4 and 5.

\section{Preliminaries}

If the vertices $i$ and $j$ of a signed graph are adjacent, then we write $i \sim j$; in particular, the existence of a positive (resp. negative) edge between these vertices is designated by $i \stackrel{+}{\sim} j$ (resp. $i \sim j$ ).

The degree of a vertex in a signed graph is equal to the number of edges incident with it. A signed graph is said to be $r$-regular if the degree of all its vertices is $r$.

The signed graphs $\dot{G}$ and $\dot{H}$ are said to be switching equivalent if there exists a set $U \subseteq V(\dot{G})$, such that $\dot{H}$ is obtained from $\dot{G}$ by reversing the sign of every edge with exactly one end in $U$. Switching equivalent signed graphs share the same underlying graph and the same spectrum. Equivalently, if the vertex labelling of $\dot{G}$ and $\dot{H}$ is transferred from their common underlying graph, then they are switching equivalent if there exists a diagonal matrix $D$ of \pm 1 s, such that $A_{\dot{H}}=D^{-1} A_{\dot{G}} D$.

A walk in a signed graph is positive if the number of its negative edges (counted with their multiplicity if there are repeated edges) is not odd. Otherwise, it is negative. In the same way we decide whether a cycle, considered as a subgraph of a signed graph, is positive or negative.

A signed doubled graph $\ddot{G}$ is obtained by doubling every edge of a graph $G$ (i.e., a signed graph with positive signature) with a negative edge. In fact, this is a signed multigraph.

We proceed by a concept of signed line graphs which is frequently used in spectral considerations, see [2, 5, 8]; a slightly different approach can be found in [10]. For a signed graph $\dot{G}$, we introduce the vertex-edge orientation $\eta: V(\dot{G}) \times E(\dot{G}) \longrightarrow\{1,0,-1\}$ formed by obeying the following rules:

(1) $\eta(i, j k)=0$ if $i \notin\{j, k\}$,

(2) $\eta(i, i j)=1$ or $\eta(i, i j)=-1$ and

(3) $\eta(i, i j) \eta(j, i j)=-\sigma(i j)$.

In fact, each edge has two orientations, and thus $\eta$ is also called a bi-orientation. The vertex-edge incidence matrix $B_{\eta}$ is the matrix whose rows and columns are indexed by $V(\dot{G})$ and $E(\dot{G})$, respectively, in such a way that its $(i, e)$-entry is equal to $\eta(i, e)$. The following relation, valid also if multiple edges exist, is well-known:

$$
B_{\eta}^{\top} B_{\eta}=2 I+A_{L(\dot{G})},
$$

where $L(\dot{G})$ is taken to be the signed line graph of $\dot{G}$. In fact, a signed line graph is a switching class, not a single signed graph, since $L(\dot{G})$ depends on orientation.

We say that a signed graph is maximal with a fixed property, if it is not an induced subgraph of a signed graph having the same property. 


\section{Root systems}

A representation of a signed graph $\dot{G}$ with least eigenvalue $\geq-2$ is a mapping $f: V(\dot{G}) \longrightarrow$ $\mathbb{R}^{k}, k \geq 1$, such that

$$
f(i) \cdot f(j)=\left\{\begin{aligned}
2 & \text { if } i=j, \\
1 & \text { if } i \pm j, \\
-1 & \text { if } i \approx j, \\
0 & \text { if } i \nsim j .
\end{aligned}\right.
$$

If $S \subset \mathbb{R}^{k}$ is the image of $f$, then we say that $\dot{G}$ is represented by $S$. Clearly, $A_{\dot{G}}+2 I$ is the Gram matrix of $S$, so it is positive semidefinite and the least eigenvalue of $\dot{G}$ is, indeed, $\geq-2$. We do not make a difference between the set $S$ and the matrix (also denoted by $S$ ) whose columns are the vectors of $S$.

If $\mathbf{e}_{\mathbf{1}}, \mathbf{e}_{\mathbf{2}}, \ldots, \mathbf{e}_{\mathbf{k}}$ are the vectors of the canonical basis of $\mathbb{R}^{k}, k \geq 4$, then the root system $D_{k}$ consists of the vectors (in the context of root systems, they are also known as the roots) $\pm \mathbf{e}_{\mathbf{i}} \pm \mathbf{e}_{\mathbf{j}}$, for $1 \leq i<j \leq k$. The root system $E_{8}$ consists of the 112 roots that also belong to $D_{8}$ and the additional 128 roots of the form $\frac{1}{2} \sum_{i=1}^{8} \pm \mathbf{e}_{\mathbf{i}}$, where the number of positive summands is not odd. The root system $E_{7}$ consists of the 126 roots of $E_{8}$ orthogonal to a fixed root $\mathrm{x} \in E_{8}$, and $E_{6}$ consists of the 72 roots of $E_{8}$ orthogonal to a fixed pair of roots $\mathbf{x}, \mathbf{y} \in E_{8}$, such that $\mathbf{x} \cdot \mathbf{y}=-1$.

A root system determines a line system, i.e., a system of one-dimensional subspaces generated by the corresponding roots. The definitions of $E_{7}$ and $E_{6}$ do not essentially depend on the choice of roots, as different choices produce isometric line systems.

For a root system $R$, a system of positive roots $R^{+}$is its subset such that:

(1) for $\pm \mathbf{x} \in R$, exactly one of $\mathbf{x},-\mathbf{x}$ belongs to $R^{+}$and

(2) for $\mathbf{x}, \mathbf{y} \in R^{+}$, if $\mathbf{x}+\mathbf{y} \in R$ then $\mathbf{x}+\mathbf{y} \in R^{+}$.

Observe that $R$ and $R^{+}$produce the same line system. In particular, we shall frequently deal with the systems of positive roots $E_{k}^{+}$(of $E_{k}$, for $6 \leq k \leq 8$ ).

It is known that every signed line graph has a representation in some $D_{k}$ [2,5]. Accordingly, the least eigenvalue of every signed line graph is $\geq-2$. A connected signed graph is said to be exceptional if it has a representation in $E_{8}$, but has not in any $D_{k}$. It follows that a connected signed graph is exceptional if and only if it is not a signed line graph, but its least eigenvalue is $\geq-2$.

For a signed graph $\dot{G}$ with $n$ vertices and least eigenvalue greater than -2 , an extension (or a (-2)-extension) of $\dot{G}$ is a signed graph with the following properties:

(1) its least eigenvalue is equal to -2 ,

(2) it contains $\dot{G}$ as an induced subgraph and

(3) exactly $n$ of its eigenvalues (with possible repetitions) are greater than -2 .

\section{Properties of exceptional signed graphs}

We start with the following lemma. 
Lemma 4.1. If a signed graph is represented by $S \subset \mathbb{R}^{k}$, then the set $S^{\prime}$ obtained by reversing the sign of some elements of $S$ represents a switching equivalent signed graph.

In particular, signed graphs represented by all the possible sets of positive roots $R^{+}$ of $R$ (where $R$ stands for any of $D_{k}, E_{6}, E_{7}, E_{8}$ ) are switching equivalent.

Proof. Given $S=\left\{\mathbf{x}_{1}, \mathbf{x}_{2}, \ldots, \mathbf{x}_{\mathbf{n}}\right\}$ and $N \subseteq\{1,2, \ldots, n\}$, let $S^{\prime}$ be obtained by reversing the sign of vectors whose indices belong to $N$. If $S$ represents a signed graph $\dot{G}$, then $S^{\prime}$ obviously represents some signed graph, say $\dot{H}$. Moreover, if $D$ is the diagonal matrix of \pm 1 s with -1 in the $i$ th position if and only if $i \in N$, then

$$
A_{\dot{G}}+2 I=S^{\top} S=D^{-1} S^{\top} S^{\prime} D=D^{-1}\left(A_{\dot{H}}+2 I\right) D=D^{-1} A_{\dot{H}} D+2 I,
$$

giving the first part of the statement.

Let $R^{+}$be a fixed set of positive roots. If $\mathbf{x}, \mathbf{y} \in R^{+}$, then $\mathbf{x} \cdot \mathbf{y} \in\{-1,0,1\}$, and so $R^{+}$ indeed represents a signed graph. In addition, any other set of positive roots is obtained by reversing the sign of some roots of $R^{+}$, and the result follows by the previous part of the proof.

The previous result can also be proved on the basis of Vijayakumar's [9, Lemma 2.3].

Throughout the remainder of the paper, we do not make a difference between switching equivalent signed graphs; in particular, when we say that some signed graph is unique (for certain property), we always mean that it is unique up to the switching equivalence.

The following 3 signed graphs play a significant role in our study: $\dot{M}_{6}$ represented by $E_{6}^{+}, \dot{M}_{7}$ represented by $E_{7}^{+}$and $\dot{M}_{8}$ represented by $E_{8}^{+}$. Each of them is unique (by Lemma 4.1). Their and spectra of the corresponding underlying graphs are given in Table 1. Note that the corresponding underlying graphs are known from literature; for example, they can be met in [3].

Table 1: Spectra of signed graphs $\dot{M}_{6}, \dot{M}_{7}$ and $\dot{M}_{8}$.

\begin{tabular}{ccc} 
Signed graph & Spectrum & Spectrum of the underlying graph \\
\hline \hline$\dot{M}_{6}$ & {$\left[10^{6},(-2)^{30}\right]$} & {$\left[20,2^{20},(-4)^{15}\right]$} \\
$\dot{M}_{7}$ & {$\left[16^{7},(-2)^{56}\right]$} & {$\left[32,4^{27},(-4)^{35}\right]$} \\
$\dot{M}_{8}$ & {$\left[28^{8},(-2)^{112}\right]$} & {$\left[56,8^{35},(-4)^{84}\right]$}
\end{tabular}

We proceed with the following theorem.

Theorem 4.2. $\dot{M}_{8}$ is the unique maximal exceptional signed graph.

Proof. If $\dot{G}$ is a maximal exceptional signed graph, then it has a representation in $E_{8}$, and consequently it has a representation in some $E_{8}^{+}$. The maximality of $\dot{G}$ implies that it is represented by the entire set of positive roots. The uniqueness follows by Lemma 4.1.

In what follows we prove a sequence of results that give a closer insight into exceptional signed graphs. We start with the following lemma and conclude the section with the forthcoming Theorem 4.8 which, together with Theorem 4.2, gives an interesting generalization of certain results concerning exceptional graphs. 
Lemma 4.3. Given $\mathbf{x}, \mathbf{x}^{\prime}, \mathbf{y}, \mathbf{y}^{\prime} \in E_{8}$, such that $\mathbf{x} \cdot \mathbf{y}=-1$ and $\mathbf{x}^{\prime} \cdot \mathbf{y}^{\prime}=-1$, let $E_{7}$ and $E_{7}^{\prime}$ stand for the sets of roots of $E_{8}$ orthogonal to $\mathrm{x}$ and $\mathrm{y}$, respectively, and let $E_{6}$ and $E_{6}^{\prime}$ stand for the sets of roots of $E_{8}$ orthogonal to the pair $\mathbf{x}, \mathbf{y}$ and $\mathbf{x}^{\prime}, \mathbf{y}^{\prime}$, respectively. The set of signed graphs with a representation in $E_{7}$ (resp. $E_{6}$ ) is equal to the set of those with a representation in $E_{7}^{\prime}$ (resp. $\left.E_{6}^{\prime}\right)$.

Proof. Let $E$ stand for one of $E_{7}$ or $E_{6}$ and $E^{\prime}$ for a corresponding $E_{7}^{\prime}$ or $E_{6}^{\prime}$.

If a signed graph $\dot{G}$ has a representation in $E$, then $A_{\dot{G}}+2 I=S^{\top} S$, for some $S \subseteq E$. Observe that an entry of $S^{\top} S$ is twice the cosine of the angle between the corresponding vectors. Since $E$ and $E^{\prime}$ determine isometric line systems, there exists $S^{\prime} \subseteq E^{\prime}$ such that $S^{\prime} \top S^{\prime}=S^{\top} S$, that is $\dot{G}$ has a representation in $E^{\prime}$.

At this point we need a result of [5]. We restate it in a slightly modified form by putting focus on relations between signed graphs in question.

Lemma 4.4. An exceptional signed graph whose least eigenvalue is greater than -2 is

(i) one of 32 signed graphs with 6 vertices,

(ii) one of 233 signed graphs with 7 vertices or

(iii) one of 1242 signed graphs with 8 vertices

listed in [5]. Every signed graph of (ii) (resp. (iii)) is a one-vertex extension of some of (i) (resp. (ii)).

In [9], Vijayakumar characterized signed graphs represented in $D_{k}$, for all $k$. In particular, the set of connected minimal forbidden subgraphs for signed line graphs has been described. It occurs that they have at most 6 vertices, and all with least eigenvalue greater than -2 are given in the previous lemma. In addition, we claim that none of them has -2 as the least eigenvalue. One may confirm this by constructing all the minimal forbidden subgraphs (by following the particular procedure described in [9]) and checking their least eigenvalue. This would be an extensive work, as just in case of graphs there are the 31 minimal forbidden subgraphs [3, p. 35]. We applied a different approach based on the computer search. Namely, we first obtained all the connected signed graphs with at most 6 vertices that are represented in $D_{6}$ and whose least eigenvalue is -2 . This procedure was performed very quickly by computer, as there are just 30 roots in $D_{6}^{+}$. Then we have just checked whether each connected signed graph with at most 6 vertices and least eigenvalue -2 (they can be found at the web page [6]) appears in the list found by computer. We record this as the following lemma.

Lemma 4.5. Every signed graph with at most 6 vertices and least eigenvalue -2 has a representation in $D_{6}$.

Here is an immediate consequence.

Corollary 4.6. Every exceptional signed graph $\dot{G}$ with least eigenvalue -2 has at least 7 vertices and contains an exceptional induced subgraph with 6 vertices and least eigenvalue greater than -2 . 
Proof. Since $\dot{G}$ is exceptional, by the mentioned result of [9], it contains a connected induced subgraph, say $\dot{H}$, with at most 6 vertices and with no representation in any $D_{k}$; in other words, $\dot{H}$ is an exceptional signed graph. By Lemma 4.5, the least eigenvalue of $\dot{H}$ is greater than -2 . By Lemma $4.4, \dot{H}$ has exactly 6 vertices and it is some of signed graphs mentioned in that lemma.

Similarly to a result concerning graphs (cf. [2]), we have the following.

Lemma 4.7. An exceptional signed graph $\dot{G}$ has between 6 and 8 eigenvalues (with possible repetitions) greater than -2 .

Proof. By Corollary 4.6, $\dot{G}$ contains an induced exceptional subgraph with 6 vertices and least eigenvalue greater than -2 , which together with the interlacing argument, gives the lower bound.

It holds $A_{\dot{G}}+2 I=S^{\top} S$, where $S$ is obtained by arranging some roots of $E_{8}$ as its columns. Since the dimension of the linear span of $E_{8}$ is 8 , the rank of $A_{\dot{G}}+2 I$ is at most 8 , giving the upper bound.

Theorem 4.8. For $6 \leq k \leq 8, \dot{M}_{k}$ is the unique maximal extension of exceptional signed graphs with $k$ vertices and least eigenvalue greater than -2 .

Proof. Every exceptional signed graph with $k(6 \leq k \leq 8)$ vertices and least eigenvalue greater than -2 has a representation in $E_{k}$, and also in $E_{k}^{+}$. Clearly, such a signed graph is an induced subgraph of a signed graph represented by the entire set of positive roots. For $k=8$, the result follows by Theorem 4.2.

For $k=7$, assuming to the contrary, we arrive at the existence of an extension, say $\dot{G}$, such that $A_{\dot{G}}+2 I=S^{\top} S$, where $S$ is obtained by arranging some roots of $E_{7}^{+}$and at least one root, say $\mathbf{x}$, of $E_{8}^{+} \backslash E_{7}^{+}$as its columns. Since $\mathbf{x} \cdot \mathbf{y}=0$, for all $\mathbf{y} \in E_{7}^{+}$, it follows that the rank of $A_{\dot{G}}+2 I$ is 8 , meaning that 8 eigenvalues (with repetitions) of $\dot{G}$ are greater than -2 , and so $\dot{G}$ cannot be an extension - a contradiction.

For $k=6$, the proof is essentially the same.

In particular case of graphs, there are exactly 473 maximal exceptional graphs, along with a large number of maximal extensions of exceptional graphs with 6 or 7 vertices, as follows by [3, Chapter 6]. On the contrary, the 'signed' generalization reported in Theorems 4.2 and 4.8 has a very simple formulation.

\section{Exceptional signed graphs with 2 eigenvalues}

In [8], we determined all the signed line graphs with 2 eigenvalues. This result is also needed here.

Theorem 5.1. A connected signed line graph has 2 eigenvalues if and only if the corresponding signed root graph is switching equivalent to

(i) the star $K_{1, n-1}$,

(ii) the negative quadrangle or the signed multigraph obtained by inserting the (parallel) negative edge between the vertices of degree 2 of the path $P_{4}$,

(iii) the complete graph $K_{n}$ or 
(iv) a signed doubled regular graph with $n$ vertices,

in all cases, for $n \geq 3$.

The next natural step is a determination of exceptional signed graphs with 2 eigenvalues. In the forthcoming Theorem 5.4, we give a characterization of such signed graphs; this result relies on Theorems 5.2 and 5.3.

If $A$ is the adjacency matrix of a signed graph with 2 eigenvalues, $\lambda$ and $\mu$, then

$$
A^{2}-(\lambda+\mu) A+\lambda \mu I=O \text {. }
$$

It follows that such a signed graph is regular with vertex degree $-\lambda \mu$.

Let $m_{\lambda}$ and $m_{\mu}$ denote the multiplicity of $\lambda$ and $\mu$, respectively, and let $t$ (resp. $q$ ) denote the difference between the numbers of positive and negative triangles (resp. quadrangles) contained.

Theorem 5.2. If $\dot{G}$ is an exceptional signed graph with 2 eigenvalues, then either $\dot{G}$ is the 3 -dimensional cube with negative quadrangles or its parameters $\left(n, r, \lambda, m_{\lambda}, \mu, m_{\mu}, t, q\right)$ have the form

$$
\left(\frac{k}{2}(\lambda+2), 2 \lambda, \lambda, k,-2, \frac{k}{2} \lambda, \frac{k}{6} \lambda\left(\lambda^{2}-4\right), \frac{k}{8} \lambda(\lambda+2)(\lambda-1)(\lambda-5)\right),
$$

where $\lambda$ is a positive integer and either

(a) $k=6,2 \leq \lambda \leq 10$,

(b) $k=7,2 \leq \lambda \leq 16$ and $\lambda$ is even or

(c) $k=8,2 \leq \lambda \leq 28$.

Proof. Let $\mu>-2$. Then either $\mu=-1$ or $\lambda=-\mu$ (as follows by considering (5.1)). In the former case, we arrive at the complete signed graph (which is non-exceptional). In the latter case, we have $r=-\lambda \mu<4$, and so $r=3$, as $r<3$ leads to non-exceptional solutions. It is known from [8] that the mentioned cube is the unique 3-regular signed graph with 2 eigenvalues $( \pm \sqrt{3})$; in addition, this is an exceptional signed graph. The last can be verified either by hand or by identifying our cube among 1242 signed graphs of Lemma 4.4(iii) (it is enumerated by 641 in the original reference).

Let $\mu=-2$. We immediately get $r=2 \lambda$, while, by virtue of Theorem 4.8 , we have $m_{\lambda}=k$, for $6 \leq k \leq 8$. By using $m_{\lambda}+m_{-2}=n$ and $\lambda m_{\lambda}-2 m_{-2}=0$, we arrive at $n=\frac{k}{2}(\lambda+2)$ and $m_{-2}=\frac{k}{2} \lambda$. Similarly, the parameters $t$ and $q$ are obtained by

$$
\begin{aligned}
m_{\lambda} \lambda^{3}-8 m_{-2} & =6 t, \\
m_{\lambda} \lambda^{4}+16 m_{-2} & =(2 r-1) r n+8 q,
\end{aligned}
$$

where the right-hand sides count the difference between the numbers of positive and negative closed walks of length 3 and 4 , respectively.

Since $\dot{G}$ is an induced subgraph of $\dot{M}_{k}$, we get the upper bounds for $\lambda$. Taking into account that all the parameters are integral if and only if either $k$ is even or $k$ is odd and $\lambda$ is even, we conclude the proof.

Here is another result giving a specified decomposition of $\dot{M}_{k}$. 
Theorem 5.3. Let $\dot{G}$ be a signed graph with a representation in $E_{k}(6 \leq k \leq 8)$ having 2 eigenvalues, $\lambda$ and -2 , and $\frac{k}{2}(\lambda+2)$ vertices. If $n$ denotes the number of vertices in $\dot{M}_{k}$ then, unless $\dot{G}$ coincides with $\dot{M}_{k}$, there exists a signed graph $\dot{H}$ with eigenvalues $\frac{2 n}{k}-\lambda-4$ (of multiplicity $k$ ) and -2 (of multiplicity $n-\frac{k}{2}(\lambda+4)$ ), such that

$$
A_{\dot{M}_{k}}=\left(\begin{array}{cc}
A_{\dot{G}} & B^{\top} \\
B & A_{\dot{H}}
\end{array}\right) .
$$

Proof. Observe that, under given assumptions, the multiplicity of $\lambda$ (in $\dot{G}$ ) is $k$. Clearly, the matrix $A_{\dot{M}_{k}}$ can be written in the form (5.2), where $A_{\dot{H}}$ is the adjacency matrix of some signed graph $\dot{H}$. If so, then by (5.1) we have $A_{\dot{H}} B=-B A_{\dot{G}}+\left(\frac{2(n-k)}{k}-2\right) B$, as the positive eigenvalue of $\dot{M}_{k}$ is $\frac{2(n-k)}{k}$. If $\mathbf{x}$ is an eigenvector associated with an eigenvalue of $\dot{G}$, then $A_{\dot{H}} B \mathbf{x}=-B A_{\dot{G}} \mathbf{x}+\left(\frac{2(n-k)}{k}-2\right) B \mathbf{x}$, giving

$$
A_{\dot{H}} B \mathbf{x}=\left(-\nu+\frac{2(n-k)}{k}-2\right) B \mathbf{x},
$$

where $\nu$ stands for either $\lambda$ or -2 .

If $B \mathbf{x}=\mathbf{0}$, then (again, by (5.1)) we have

$$
A_{\dot{M}_{k}}\left(\begin{array}{c}
\mathbf{x} \\
\mathbf{0}
\end{array}\right)=\left(\begin{array}{c}
A_{\dot{G}} \mathbf{x} \\
\mathbf{0}
\end{array}\right)=\nu\left(\begin{array}{l}
\mathbf{x} \\
\mathbf{0}
\end{array}\right) .
$$

The case $\nu=\lambda$ cannot occur in (5.4), since $\dot{M}_{k}$ and $\dot{G}$ cannot share the same eigenvalues because they differ in vertex degree. Therefore, $\frac{2 n}{k}-\lambda-4$ is an eigenvalue of $\dot{H}$, with multiplicity at least $k$. Moreover, its multiplicity must be exactly $k$, as follows by interchanging the roles of $\dot{G}$ and $\dot{H}$ and considering (5.3) (with $A_{\dot{G}}$ instead of $A_{\dot{H}}$ and $B^{\top}$ instead of $B$ ); a larger multiplicity would imply either $\lambda=\frac{2(n-k)}{k}$ or $\lambda=-2$, both impossible.

Since $\dot{H}$ has $n-\frac{k}{2}(\lambda+2)$ vertices, there are exactly $n-\frac{k}{2}(\lambda+4)$ remaining eigenvalues (with repetitions). If $n-\frac{k}{2}(\lambda+4)=0$, then $\dot{H}$ is totally disconnected (with $k$ vertices), and we are done; this is a degenerate case where $\dot{H}$ has only one eigenvalue. Otherwise, if $\bar{d}$ is the average value of the remaining eigenvalues, then by

$$
k\left(\frac{2 n}{k}-\lambda-4\right)+\left(n-\frac{k}{2}(\lambda+4)\right) \bar{d}=0,
$$

we get $\bar{d}=-2$, which (since $\dot{H}$ is an induced subgraph of $\dot{M}_{k}$ ) implies that -2 is the unique remaining eigenvalue with the desired multiplicity.

In the light of the previous result, we say that $\dot{M}_{k}$ is decomposed into $\dot{G}$ and $\dot{H}$. We are now in position to give the following characterization.

Theorem 5.4. If $\dot{G}$ is an exceptional signed graph with 2 eigenvalues, then either

(i) $\dot{G}$ is the 3-dimensional cube with negative quadrangles, $\dot{M}_{6}, \dot{M}_{7}, \dot{M}_{8}$ or

(ii) the parameters of $\dot{G}$ are determined by Theorem 5.2 and $\dot{G}$ is obtained from a decomposition of $\dot{M}_{k}$ in sense of Theorem 5.3. 
The proof follows directly. Observe that if $\dot{M}_{k}$ is decomposed as in Theorem 5.3, then a special case where $\dot{G}$ and $\dot{H}$ are isomorphic (possibly, after an appropriate switching) may occur. Here are some particular cases:

- Besides the cube described in Theorem 5.2, in [8] (but see also [4, 7]) the existence of the 2 additional exceptional signed graphs with 2 eigenvalues is confirmed; they correspond to data of Theorem 5.2 obtained for $(k, \lambda)=(7,2)$ and $(k, \lambda)=(8,2)$, respectively.

- Setting $(k, \lambda)=(7,14)$ and $(k, \lambda)=(8,26)$ in Theorem 5.2, we get that $\dot{G}$ is paired (in sense of Theorem 5.3) with a totally disconnected graph with 7 and 8 vertices, respectively. Since $\dot{M}_{k}$ contains a co-clique with the corresponding number of vertices, we may confirm the existence of $\dot{G}$; clearly, it is exceptional.

Note that every signed graph represented by all the non-integral roots of $E_{k}^{+}$, for $6 \leq k \leq 8$, is exceptional. Therefore, every signed graph represented by these and possibly some other roots of $E_{k}^{+}$is also exceptional.

- Up to the switching equivalence, $L\left(K_{8}\right)$ is represented by the roots $\mathbf{e}_{\mathbf{i}}-\mathbf{e}_{\mathbf{j}}(1 \leq i<$ $j \leq 8)$. Thus, it has a representation in $E_{8}^{+}$; moreover, since all the corresponding roots are orthogonal to $\frac{1}{2} \mathbf{j} \in E_{8}^{+}, L\left(K_{8}\right)$ has a representation in $E_{7}^{+}$, as well. It is paired with the signed graph with $(k, \lambda)=(7,8)$, which is exceptional by the previous observation.

To get another example we need the following theorem.

Theorem 5.5. If $F$ is a regular graph with 8 vertices, then $L(\ddot{F})$ is represented by integral roots of $E_{8}^{+}$.

Proof. Since each column of the vertex-edge incidence matrix $B_{\eta}$ of $\ddot{F}$ has length 8 and contains exactly 2 non-zero entries, it follows that $L(\ddot{F})$ has a representation in $D_{8}^{+}$, which is sufficient to conclude the proof.

- If $F$ is $r$-regular with $k$ vertices, then by (5.1) the spectrum of $L(\ddot{F})$ is

$$
\left[2(r-1)^{k},(-2)^{k(r-1)}\right]
$$

and so, for $k=8, L(\ddot{F})$ is paired with an exceptional signed graph with spectrum $\left[2(14-r)^{8},(-2)^{8(14-r)}\right]$. Its existence covers many particular cases of Theorem 5.2 .

We continue by some non-existences.

Theorem 5.6. There is no exceptional signed graph neither for $k=6$ and $\lambda \notin\{4,5,10\}$ nor $(k, \lambda)=(8,27)$, where the parameters $k$ and $\lambda$ are determined in the formulation of Theorem 5.2 .

Proof. Denote the putative signed graph by $\dot{G}$, and let $\dot{H}$ be the other signed graph of a decomposition described in Theorem 5.3.

The case $(k, \lambda)=(6,2)$ cannot occur, since the only connected signed graph with the corresponding spectrum is $L\left(\ddot{C}_{6}\right)$ [8], so non-exceptional. 
The case $(k, \lambda)=(6,3)$ is eliminated by the computer search.

For $(k, \lambda)=(6,6)$ and $(k, \lambda)=(6,7), \dot{H}$ would be the mentioned $L\left(\ddot{C}_{6}\right)$ and the disconnected signed graph consisting of the 3 negative triangles, respectively, but this is impossible since these signed graphs have no representation in $E_{6}$.

For $(k, \lambda)=(6,8), \dot{H}$ would be totally disconnected with 6 vertices, but this is impossible since the size of the largest co-clique in $\dot{M}_{6}$ is 4 .

The case $(k, \lambda) \in\{(6,9),(8,27)\}$ is resolved easily on the basis of Theorem 5.3 ( $\dot{H}$ does not exist).

Therefore, $\dot{G}$ does not exist.

We conclude the section with the 'line' counterparts to Theorem 4.2 and Lemma 4.4.

Theorem 5.7. If $\dot{G}$ is a signed line graph with a representation in $D_{k}$ (for some fixed $k$ ), then $\dot{G}$ is an induced subgraph of $L\left(\ddot{K}_{k}\right)$.

Proof. This follows since $L\left(\ddot{K}_{k}\right)$ is represented by all the roots of $D_{k}^{+}$.

We believe that the reader is familiar with the concept of star complements in signed graphs, where an induced subgraph $\dot{H}$ is said to be a star complement in its $k$-vertex extension $\dot{G}$ for an eigenvalue $\nu$ if $\nu$ is not an eigenvalue of $\dot{H}$, but is an eigenvalue of $\dot{G}$ with multiplicity $k$. For more details, see [3]. A connected signed multigraph with equal number of vertices and edges is called unicyclic.

Lemma 5.8. For every connected $r$-regular graph $F$ with vertex degree at least $2, \ddot{F}$ contains a unicyclic subgraph whose signed line graph is a star complement for -2 in $L(\ddot{F})$.

Proof. By (5.5), the multiplicity of -2 in $L(\ddot{F})$ is $t(r-1)$ ( $t$ being the number of vertices in $F$ ), and thus there exists a star complement, say $\dot{H}$, for -2 , where $\dot{H}$ has $t r-t(r-1)=t$ vertices. By [1], it can be chosen to be connected. Then, its signed root multigraph is also connected and has $t$ edges, so it is unicyclic.

A cycle in $\ddot{F}$ can be formed by 2 parallel edges, as well.

By Lemmas 4.4 and 4.7, if $\dot{H}$ is an exceptional star complement for -2 (in some exceptional signed graph), then $\dot{H}$ is one of signed graphs listed in the former lemma. By following the proof of Lemma 5.8, one can obtain star complements in signed line graphs of Theorem 5.1(i)-(iii); in fact, this is a matter of routine. In this way, we have described the basis of all star complements for -2 in signed graphs with 2 eigenvalues.

\section{ORCID iD}

Zoran Stanić (D) https://orcid.org/0000-0002-4949-4203

\section{References}

[1] F. Belardo, E. M. Li Marzi and S. K. Simić, Signed line graphs with least eigenvalue -2 : the star complement technique, Discrete Appl. Math. 207 (2016), 29-38, doi:10.1016/j.dam.2016. 02.018 .

[2] P. J. Cameron, J.-M. Goethals, J. J. Seidel and E. E. Shult, Line graphs, root systems, and elliptic geometry, J. Algebra 43 (1976), 305-327, doi:10.1016/0021-8693(76)90162-9. 
[3] D. Cvetković, P. Rowlinson and S. Simić, Spectral Generalizations of Line Graphs: On Graphs with Least Eigenvalue -2, volume 314 of London Mathematical Society Lecture Note Series, Cambridge University Press, Cambridge, 2004, doi:10.1017/cbo9780511751752.

[4] E. Ghasemian and G. H. Fath-Tabar, On signed graphs with two distinct eigenvalues, Filomat 31 (2017), 6393-6400, doi:10.2298/fil1720393g.

[5] G. Greaves, J. Koolen, A. Munemasa, Y. Sano and T. Taniguchi, Edge-signed graphs with smallest eigenvalue greater than -2, J. Comb. Theory Ser. B 110 (2015), 90-111, doi:10.1016/ j.jctb.2014.07.006.

[6] Z. Stanić, Signed graphs of small order, http://www.math.rs/ zstanic/siggr. htm.

[7] Z. Stanić, Integral regular net-balanced signed graphs with vertex degree at most four, Ars Math. Contemp. 17 (2019), 103-114, doi:10.26493/1855-3974.1740.803.

[8] Z. Stanić, Spectra of signed graphs with two eigenvalues, Appl. Math. Comput. 364 (2020), 124627 (9 pages), doi:10.1016/j.amc.2019.124627.

[9] G. R. Vijayakumar, Signed graphs represented by $D_{\infty}$, European J. Combin. 8 (1987), $103-$ 112, doi:10.1016/s0195-6698(87)80024-0.

[10] T. Zaslavsky, Matrices in the theory of signed simple graphs, in: B. D. Acharya, G. O. H. Katona and J. Nešetřil (eds.), Advances in Discrete Mathematics and Applications: Mysore, 2008, Ramanujan Mathematical Society, Mysore, volume 13 of Ramanujan Mathematical Society Lecture Notes Series, 2010 pp. 207-229, Proceedings of the International Conference (ICDM 2008) held at the University of Mysore, Mysore, June 6 - 10, 2008. 